\title{
EFEITO CINÉTICO DE COMPENSAÇÃO OBSERVADO NA REAÇÃO DE CURA DE RESINA EPÓXI (DGEBA) COM DICIANODIAMIDA
}

\author{
P. E. FERREIRA ${ }^{1}$, J. G. R. POÇO ${ }^{2,3}$ \\ ${ }^{1}$ Trabalho Final apresentado ao IPT para obtenção do Mestrado Profissional em Processos Industriais \\ ${ }^{2}$ Centro Universitário da FEI - Depto. de Eng ${ }^{\mathrm{a}}$. Quimica \\ ${ }^{3}$ IPT - Instituto de Pesquisas Tecnológicas do Estado de São Paulo S/A \\ E-mail para contato: jgrpoco@ fei.edu.br; jgrpoco@ipt.br
}

\begin{abstract}
RESUMO - A cinética de reação de uma resina epóxi do tipo diglicidil éter de bisfenol-A (DGEBA) curada com dicianodiamida foi estudada por meio da técnica de calorimetria exploratória diferencial (DSC) usando quantidades estequiométricas dessas substâncias e preparadas como uma base para tinta em pó. Os dados foram obtidos experimentalmente por meio de ensaios dinâmicos, com diferentes taxas de aquecimento, ou isotérmicos, a diferentes temperaturas. Foram aplicados os modelos cinéticos de Borchardt e Daniels e isotérmico de ordem n, para determinação dos parâmetros cinéticos da reação (Ea, ko) usando software conveniente. Observou-se que com uso da dicianodiamida como catalisador, todas as energias de ativação foram diferentes entre si. O fenômeno cinético de compensação, caracterizado pela linearidade mostrada pelos parâmetros $\left(\mathrm{E}_{\mathrm{a}} \mathrm{e} \mathrm{k}_{0} \mathrm{ou} \mathrm{Z}\right)$ no diagrama de Cremer-Constable indicou a existência de mecanismos e ordens de reação diferentes, fato que dificulta a previsão do tempo de cura.
\end{abstract}

\section{INTRODUÇÃO}

Uma resina epóxi pode ser definida como um composto líquido, sólido ou pastoso que possui dois ou mais grupos 1,2-epóxi (ou também chamados grupos glicidila). A resina (DGEBA - diglicidil éter de bisfenol-A) é sintetizada pela reação do bisfenol-A, composto resultante da combinação de acetona com dois grupos fenólicos, e a epicloridrina, a qual é produzida a partir de propileno e cloro (FAZENDA, 1995, vol.1, cap.5). As resinas do tipo DGEBA apresentam boa flexibilidade e resistência química. A estrutura química deste produto é apresentada na Figura 1. O processo de cura em sistemas epoxídicos envolve uma reação dos grupos epóxi terminais da resina com os grupos ativos ou funcionais do endurecedor. A molécula de dicianodiamida é apresentada na Figura 2. A molécula de dicianodiamida contém 3 grupos com nitrogênio e hidrogênio que podem reagir com um anel epoxídico

As principais reações envolvidas na cura de uma resina epóxi com endurecedor amínico podem ser representadas pelo mecanismo apresentado na Figura 3. 
<smiles>CC(C)(COc1ccc(C(C)(C)c2ccc(OCC(O)COc3ccc(C(C)(C)c4ccc(OCC5CO5)cc4)cc3)cc2)cc1)c1ccc(OCC2CO2)cc1</smiles>

Figura 1 - Estrutura química de uma resina epóxi tipo DGEBA<smiles>N#CNC(=N)N</smiles>

Figura 2 - Estrutura química da dicianodiamida (FAZENDA,1995).<smiles>[R]NCCC(O)CN([2H])C</smiles>

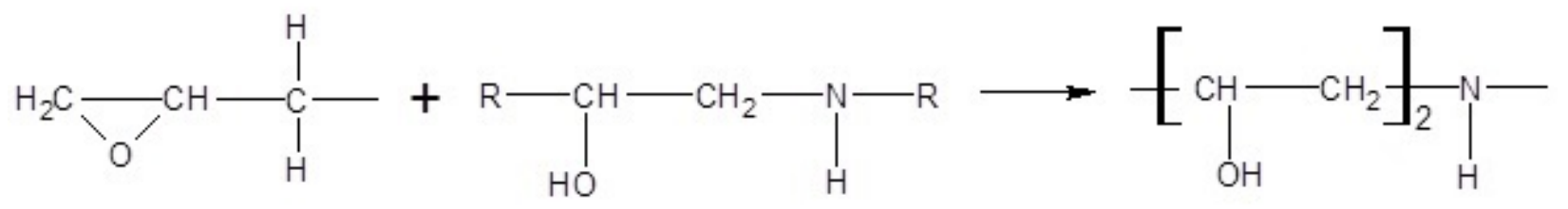

Figura 3 - Mecanismo de cura epóxi-amina (FLAMMERSHEIM, 1998)

O mecanismo de cura é complexo, envolvendo reações de adição tanto com o anel epoxídico quanto com o grupo hidroxila existente na molécula de resina epóxi, e simultaneamente essas reações catalisam a reação entre dois grupos epóxi (FAZENDA,1995). A complexidade do mecanismo de reação faz com que a cura com dicianodiamida não siga uma estequiometria exata, e a baixa solubilidade na resina epóxi tende a influenciar a cinética da reação. Uma maior temperatura de cura auxilia na dissolução da dicianodiamida, assim, facilita a reação desta com epóxi e aumenta a velocidade da reação. (HONG \& CHANG, 2004). O sistema epóxi-dicianodiamida curado consiste principalmente de uma eterificação favorecida a baixas temperaturas e uma reação epóxi-amina favorecida a altas temperaturas (LIN et al, 1986, apud HONG \& CHANG, 2004).

O chamado efeito cinético de compensação consiste numa interrelação entre os valores da energia de ativação e o fator de freqüência de uma reação, onde uma mudança em um destes 
parâmetros é compensada pela mudança no outro (POÇO, FURLAN, GIUDICI, 2001). A interrelação entre a energia de ativação e o fator de frequiência é caracterizada pela Equação 1 podendo também ser colocada na forma conhecida como relação de Cremer-Constable (Equação 2):

$$
\begin{aligned}
& \ln Z=a E+b \\
& \ln Z=\ln k_{i}+\frac{E_{a}}{R} \cdot \frac{1}{T_{i}}
\end{aligned}
$$

onde $\mathrm{k}_{\mathrm{i}}$ é conhecida como constante isocinética $\left(\mathrm{b}=\ln \mathrm{k}_{\mathrm{i}}\right)$, é a temperatura isocinética ou de compensação $\left(\mathrm{a}=1 /\left(\mathrm{RT}_{\mathrm{i}}\right)\right.$. Segundo GALWEY (1977, apud GALWEY, 2004), a temperatura isocinética representa a temperatura onde, em um mecanismo complexo, as reações do sistema apresentam o mesmo valor para a constante de velocidade $(\mathrm{k}(\mathrm{T}))$. O efeito de compensação é um padrão de comportamento cinético largamente reportado e para o qual ainda não existe uma explicação teórica aceita (GALWEY \& BROWN, 1997). O efeito de compensação é, portanto, identificado pela linearidade dos valores do fator de frequiência e da energia de ativação na relação descrita pela equação (1).

KOGA (1994) classifica os tipos de efeito de compensação entre parâmetros cinéticos obtidos via análise térmica em três diferentes categorias:

- propriedades físico-químicas da amostra: variação nas propriedades da amostra gera um efeito de compensação empírico e difícil de ser quantificado.

- procedimento experimental: mudanças nas condições experimentais utilizadas, como massa da amostra e taxa de aquecimento, geram um efeito de compensação.

- modelo cinético envolvido: parâmetros cinéticos calculados utilizando-se modelos inadequados apresentam efeito de compensação.

O objetivo do presente trabalho é o estudo da reação de cura da resina resina epóxi (DGEBA) com dicianodiamida por calorimetria difencial exploratória usando os modelos cinéticos mais comuns presentes nos softwares. Outrossim, pretende-se mostrar que esses modelos são inadequados quando a reação apresenta o efeito cinético de compensação.

\section{PARTE EXPERIMENTAL}

A parte experimental do presente consistiu na e avaliação da cura por calorimetria exploratória diferencial de amostras de tinta em pó obtida a partir de uma resina epóxi do tipo DGEBA fabricada pela Dow Química (DER 664U) e o endurecedor selecionado, a dicianodiamida fabricada pela Huntsman (Aradur). As amostras do sistema estudado foram submetidas à calorimetria exploratória diferencial em um equipamento da marca Metler Toledo modelo DSC 822e, acoplada a uma unidade de resfriamento com gás freon (intracooler). As técnicas empregadas foram as de experimentos dinâmicos na qual aplicou-se taxas constante de aquecimento de 5, 10, 15 e $20{ }^{\circ} \mathrm{C}$ por minuto, e 
experimentos isotérmicos, realizada à temperaturas constantes de, com pré-aquecimento da célula de DSC na temperatura selecionada utilizando de 180, 190, 200 e $210{ }^{\circ} \mathrm{C}$. Em todos experimentos foi utilizado nitrogênio como gás de purga. Antes de iniciar os experimentos, o equipamento foi aferido com padrões de índio e chumbo.

Os dados obtidos foram analisados por meio de modelos cinéticos bastante conhecidos tais como os modelos de Borchardt \& Daniels, baseado em cinética de ordem n. O detalhamento experimental completo encontra-se publicado (FERREIRA, 2005).

\section{RESULTADOS E DISCUSSÃO}

\subsection{Experimentos dinâmicos}

As curvas obtidas nos experimentos dinâmicos foram avaliadas com o software STARe, doisponível no equipamento, o qual fornece diretamente características da reação como entalpia e temperatura de pico, além de parâmetros cinéticos como energia de ativação e ordem de reação.

A figura 4 apresenta as curvas dinâmicas exotérmicas obtidas correspondentes à reação de cura via calorimetria exploratória diferencial para o sistema epóxi-dicianodiamida. Os calores de reação exotérmicos obtidos nos experimentos dinâmicos são apresentados na Tabela 1.

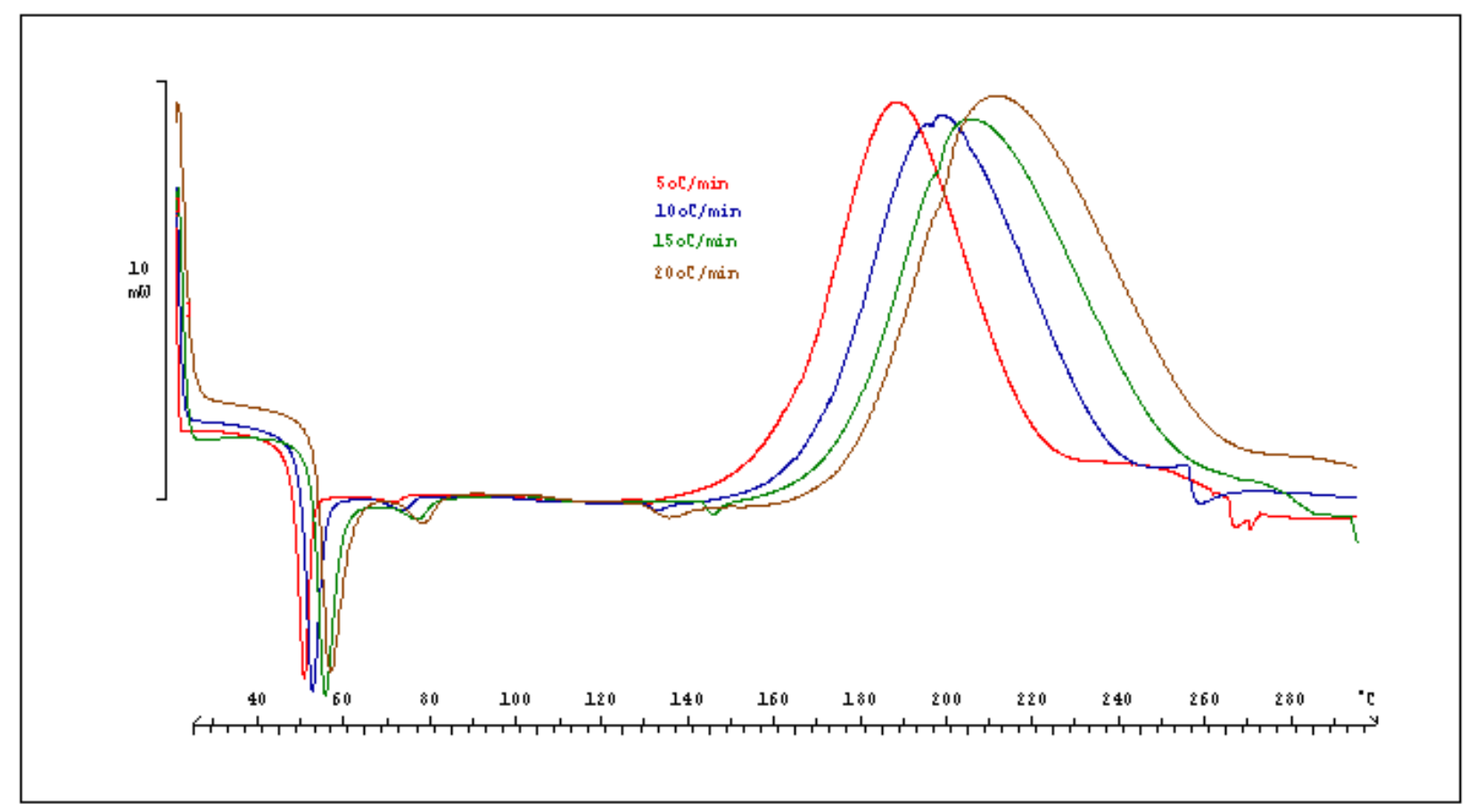

Figura 4 - Curvas obtidas via experimentos dinâmicos com DSC

Os dados obtidos mostram que a reação se inicia ("onset") em temperaturas na faixa de 170 a 
$190{ }^{\circ} \mathrm{C}$, o que está de acordo com a literatura sobre sistemas epóxi curados com dicianodiamida. A energia liberada pela reação pode ser considerada independente da razão de aquecimento a qual a amostra é submetida, porém pode-se observar que com a elevação da taxa de aquecimento a reação passa a iniciar-se a uma temperatura mais alta.

Tabela 1 - Calor de reação obtidos nos experimentos

\begin{tabular}{|c|c|}
\hline Taxa de aquecimento $\left({ }^{\circ} \mathrm{C} / \mathrm{min}\right)$ & Calor de reação $(\mathrm{J} / \mathrm{g})$ \\
\hline 5 & 117 \\
\hline 10 & 119 \\
\hline 15 & 124 \\
\hline 20 & 119 \\
\hline
\end{tabular}

Determinação de parâmetros cinéticos pelo método de Borchardt e Daniels. A Tabela 2 apresenta os valores de energia de ativação, ordem de reação e fator pré-exponencial para os experimentos realizados, fornecidos pelo software STARe de análise térmica. O modelo utilizado é o de Borchardt e Daniels (cinética de ordem n).

Tabela 2 - Parâmetros cinéticos obtidos com o modelo de Borchardt e Daniels

\begin{tabular}{|c|c|c|c|}
\hline Taxa $\left({ }^{\circ} \mathrm{C} / \mathrm{min}\right)$ & $\mathrm{E}_{\mathrm{a}}(\mathrm{kJ} / \mathrm{mol})$ & Ordem da reação $(-)$ & Fator pré-exponencial $(\ln \mathrm{Z})$ \\
\hline 5 & 177 & 1,77 & 40,79 \\
\hline 10 & 154 & 1,75 & 34,56 \\
\hline 15 & 138 & 1,78 & 30,04 \\
\hline 20 & 127 & 1,70 & 27,21 \\
\hline
\end{tabular}

Segundo os dados obtidos, a reação possui ordem entre 1,70 e 1,78. Observa-se também uma de uma variação dos parâmetros cinéticos energia de ativação e fator pré-exponencial com a taxa de aquecimento.

O comportamento da energia de ativação ao variar-se a taxa de aquecimento é avaliado na Figura 5, onde estão plotados os valores de energia de ativação (Ea) e logaritmo do fator exponencial ( $\ln \mathrm{Z}$ ou $\ln \mathrm{k}_{0}$ ), num diagrama conhecido como Cremer-Constable. O gráfico anterior mostra que a existência bem definida do efeito cinético de compensação, o que explica os diferentes valores de energia de ativação e fator pré-exponencial encontrados nas curvas dinâmicas

O gráfico anterior mostra que a existência bem definida do efeito cinético de compensação, o que explica os diferentes valores de energia de ativação e fator pré-exponencial encontrados nas curvas dinâmicas. Os valores obtidos via regressão linear para as constantes a e b foram de 0,2745 e 7,7942, respectivamente. 


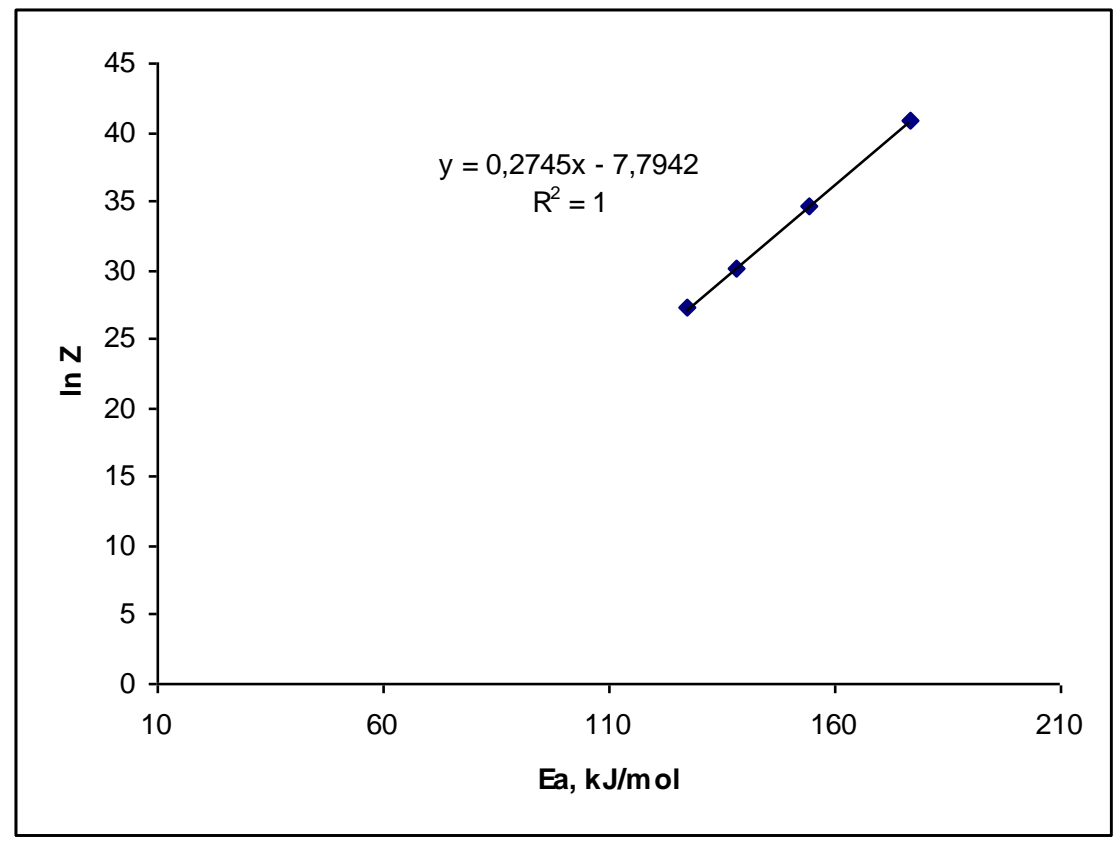

Figura 5 - Efeito cinético de compensação observado pelo Diagrama de Cremer-Constable

\subsection{Experimentos isotérmicos}

As curvas obtidas por meio dos experimentos isotérmicos apresentam velocidade máxima no instante de tempo igual a zero, indicando que o mecanismo de reação segue o modelo de ordem $\mathrm{n}$. O comportamento das curvas isotérmicas obtidas, no que se refere à posição do pico máximo, não obedece ao exposto na literatura sobre reações de cura epóxi-amina. Entretanto alguns trabalhos sobre a cura de resinas epóxi com dicianodiamida, como HONG \& WU (1998), reportam comportamentos muito semelhantes para as curvas obtidas com experimentos isotérmicos.

Os dados calculados pelo software STARe, são apresentados na Tabela 3 para os experimentos isotérmicos. Os calores de reação são comparáveis aos obtidos nos experimentos dinâmicos. A variação na ordem da reação pode ser atribuída à maior dissolução da dicianodiamida em temperaturas mais elevadas, como é relatado por HONG \& WU (1998) e HONG \& CHAN (2004). Nos experimentos dinâmicos, devido ao aumento gradativo na temperatura, as diferenças de solubilidade da dicianodiamida entre as diferentes taxas de aquecimento empregadas podem ser negligenciadas.

A energia de ativação e o fator pré-exponencial foram calculados pelo gráfico de Arrhenius, plotando-se $\ln k(T)$ em função de $1 / T$. Os calores de reação são comparáveis aos obtidos nos experimentos dinâmicos. A variação na ordem da reação pode ser atribuída à maior dissolução da dicianodiamida em temperaturas mais elevadas, como é relatado por HONG \& WU (1998) e HONG \& CHAN (2004). Nos experimentos dinâmicos, devido ao aumento gradativo na temperatura, as diferenças de solubilidade da dicianodiamida entre as diferentes taxas de aquecimento empregadas podem ser negligenciadas. 
Tabela 3 - Dados obtidos nos experimentos isotérmicos

\begin{tabular}{|c|c|c|c|}
\hline Temperatura $\left({ }^{\circ} \mathrm{C}\right)$ & Calor de reação $(\mathrm{J} / \mathrm{g})$ & Ordem $(-)$ & $\ln \mathrm{k}(\mathrm{T})$ \\
\hline 180 & 118,42 & 0,73 & $-5,92$ \\
\hline 190 & 114,36 & 0,83 & $-5,28$ \\
\hline 200 & 122,85 & 0,94 & $-4,87$ \\
\hline 210 & 118,85 & 1,00 & $-4,53$ \\
\hline
\end{tabular}

Os calores de reação são comparáveis aos obtidos nos experimentos dinâmicos. A variação na ordem da reação pode ser atribuída à maior dissolução da dicianodiamida em temperaturas mais elevadas, como é relatado por HONG \& WU (1998) e HONG \& CHAN (2004). Nos experimentos dinâmicos, devido ao aumento gradativo na temperatura, as diferenças de solubilidade da dicianodiamida entre as diferentes taxas de aquecimento empregadas podem ser negligenciadas.

A energia de ativação e o fator pré-exponencial foram calculados pela análise de Arrhenius, plotando-se ln k (T) em função de 1/T. Os valores calculados para os parâmetros de Arrhenius para os experimentos isotérmicos foram $\mathrm{E}_{\mathrm{a}}=83,52 \mathrm{~kJ} / \mathrm{mol}$ e $\ln \mathrm{Z}=16,33$. Os valores obtidos também se encontram alinhados com aqueles obtidos nos experimentos dinâmicos no diagrama de CremerConstable, e portanto sob o efeito de compensação.

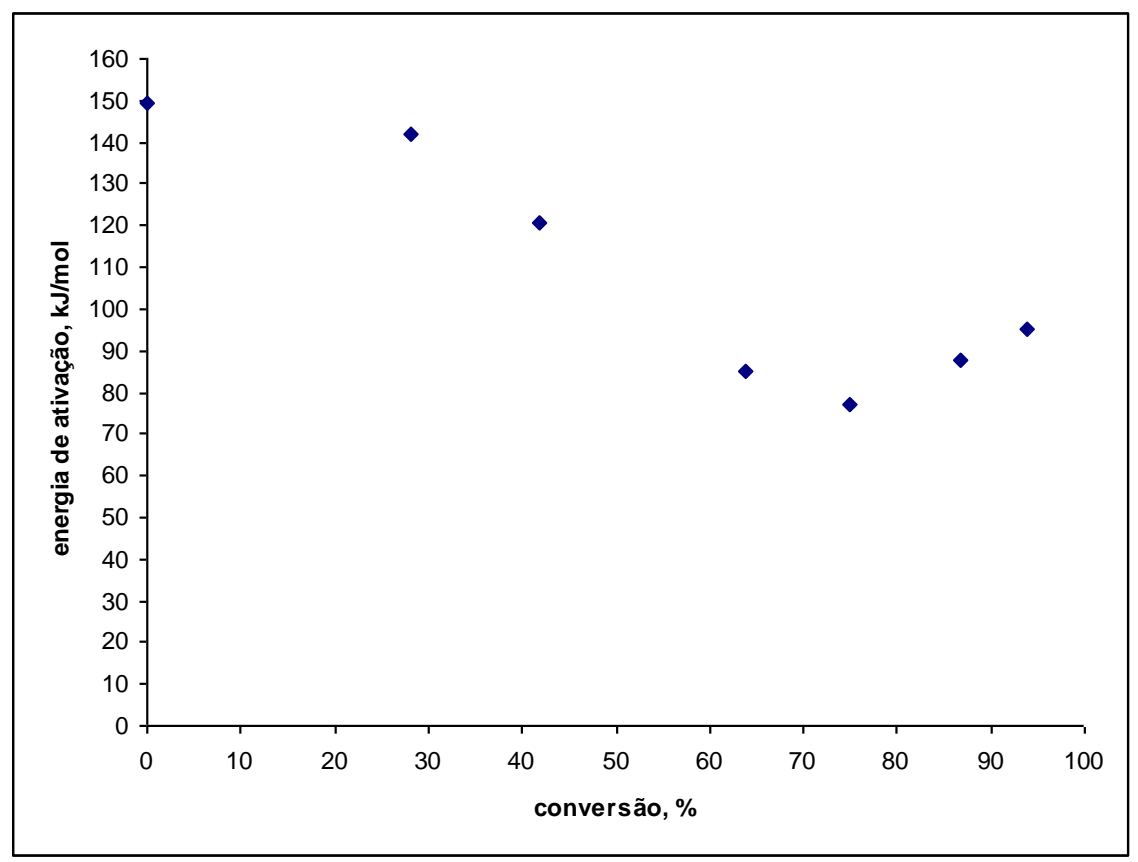

Figura 6 - Relação entre a energia de ativação e conversão da reação para cura isotérmica a $180{ }^{\circ} \mathrm{C}$ do sistema epóxi-dicianodiamida

FERREIRA (2005) observou que apenas a partir dos dados cinéticos obtidos (Ea e ln Z) obtidos nos experimentos isotérmicos se obtém uma boa previsão do tempo de cura do sistema estudado. 


\section{9 a 22 de outubro de 2014 \\ Florianópolis/SC}

Observou ainda em experimentos específicos (FERREIRA, 2005) que energia de ativação varia em função da conversão conforme mostrado na Figura 6 e que os pares de parâmetros cinéticos também se encontram alinhados com os demais valores obtideos no Diagrama de Cremer-Constable. Isso mostra que o mecanismo de reação varia conforme a conversão. Essa variação pode estar associada a existência de mais de um centro ativo na molécula de dicianodiamina.

\section{Conclusões}

A partir dos dados obtidos nos experimentos dinâmicos pelo modelo de Borchardt e Daniels não foi possível obter parâmetros cinéticos $\left(E_{a}\right.$ e $\left.\ln Z\right)$ que representasse bem o desempenho de cura do sistema estudado. Apenas com os valores obtidos de $\left(E_{a}\right.$ e $\left.\ln Z\right)$ a partir dos experimentos isotérmicos foi possivel representar o sistema. Isso ocorre porque o sistema encontra-se sobre o efeito cinético de compensação demosntrado pelo alinhamento dos pares de valores dos parâmetros cinéticos no diagrama de Cremer-Constable. E a origem desse fenômeno estaria associada a existência de mais de um centro ativo na molécula de dicianodiamina que apresenta três grupos com nitrogênio e hidrogênio com caracterísiticas diferentes que podem reagir com um anel epoxídico de forma diferente e dependente da conversão.

\section{Referências bibliográficas}

FAZENDA, J.M.R. Resinas epoxídicas. In: Tintas \& Vernizes Ciência e Tecnologia. São Paulo: Associação Brasileira dos Fabricantes de Tintas (ABRAFATI), 1995. Vol.1, cap. 5.

FERREIRA, P.E. Estudo cinético da reação de cura em sistemas poliméricos baseados em resina epóxi. Dissertação de mestrado profissional do IPT, São Paulo, 2005.EY, A., Is the science of thermal analysis kinetics based on solid foundations? A literature appraisal. Thermochimica Acta, 413, p139183, 2004.

FLAMMERSHEIM, H.J. Kinetics and mechanism of the epoxide-amine polyaddition. Thermochimica Acta, 310, p153-159, 1998.

GALWEY, A. \& BROWN, M. E., Arrhenius parameters and compensation behaviour in solid-state decompositions. Thermochimica Acta, 300, p107-115, 1997.

HONG, S.G. \& CHAN, C.K. The curing behaviors of the epoxy/dicyandiamide system modified with epoxidized natural rubber. Thermochimica Acta, 417, p99-106, 2004.

HONG, S.G \& WU, C.S. DSC and FTIR analysis of the curing behaviors of epoxy/SICY/solvent open systems. Thermochimica Acta, 316, p167-175, 1998.

KOGA, N., A review of the mutual dependence of Arrhenius parameters evaluated by thermoanalytical study of solid-state reactions: the kinetic compensation effect. Thermochimica Acta, 244, p1-20, 1994.

POÇO, J.G.R., FURLAN, H., GIUDICI, R. A Discussion on Kinetic Compensation Effect and Anisotropy. J. Phys Chem B, 106 (18), pp 4873-4877, 2002. 